\title{
Is Overtriage Associated With Increased Mortality? The Evidence Says "Yes"
}

John H. Armstrong, MD, FACS, Jeffrey Hammond, MD, MPH, FACS, Asher Hirshberg, MD, FACS, and Erik R. Frykberg, MD, FACS

Things should be made as simple as possible, but not any simpler.-Albert Einstein

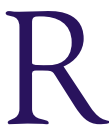
esearch in disaster medicine in general, and triage in particular, is largely descriptive and conducted after the fact because disasters do not lend themselves to study via randomized controlled trials. A useful alternative involves modeling and analysis based on parameters obtained from real disasters. Triage is particularly appropriate for this kind of analysis because it involves a relatively distinct problem space, with discrete decisions made at well-defined points in time. Defining and varying the inputs into particular triage schemes should therefore permit model predictions across a variety of scenarios. In particular, the effects of overtriage, or labeling noncritical casualties as critical, could be demonstrated relative to critical mortality, or the mortality of critical casualties with an Injury Severity Score (ISS) of $>15$. The results of this modeling could then be applied to planning and practice.

Examining the effect of overtriage on critical mortality, Hupert and colleagues have modeled triage as a diagnostic test that gives a result of critical or noncritical to casualties in sequence; this result then leads to prioritized resource utilization. ${ }^{1}$ The model emphasizes trauma system capability, rather than individual hospital capability. Modeling triage in this way allowed the authors to vary not only triage sensitivity but also the prevalence of critical casualties and hence to examine the effects of overtriage across a range of hypothetical casualty loads.

Using this modeling approach, Hupert et al claim to demonstrate that overtriage had minimal effect on critical mortality. Variation in the prevalence of critical casualties showed, not surprisingly, that critical mortality increased when higher overtriage resulted from adding more noncritical casualties to the case mix, and that critical mortality decreased when there were fewer critical casualties. Treatment capability had a larger effect on outcomes than triage performance.

When trying to assess the usefulness of a model, however, a key question is whether its structure approximates or reflects the clinical reality. Simulation using modeling is the imitation of a real-world process that can lead to inferences about the operating characteristics of the real system. As such, the fidelity and validity of the model are dependent upon the accuracy of the underlying assumptions and logic. The model of Hupert et al deviates from the real-life experience of trauma systems dealing with mass casualty incidents in three important respects: it assumes an orderly sequential flow of casualties, which almost never occurs in real mass casualty incidents ${ }^{2}$; it links the trauma bay in the emergency department with an operating room, even though only a small minority of critical casualties in a real $\mathrm{MCI}$ require surgery ${ }^{3}$; and it uses the START triage algorithm that is mostly applicable to field situations and almost irrelevant to triage at the hospital door.

The model does, in fact, show a positive correlation between overtriage and critical mortality when the number of noncritical casualties increases. This observation is compatible with reported experience. Evidence derived from urban terrorism during the past 40 years clearly shows that overtriage is directly related to critical mortality (Fig 1). More recent urban disasters show better tolerance of overtriage, compared with earlier or rural events, but at some inevitable point, the system is overwhelmed, and the mortality of people who are critically injured rises regardless of the setting. The factors responsible for this apparent improvement in tolerating the adverse effects of overtriage are unclear. Nevertheless, the message emerging from analysis of real-life data, as opposed to theoretical models, remains that at some point, the ability to provide optimal care will be overcome by the casualty load. Noncritical casualties who are erroneously sorted as critical increase the "critical casualty" workload while competing for limited trauma-care resources, assets, and expertise with genuine critical casualties.

An important aspect of any scientific discussion of triage is that the sorting process does not involve a single decision point but rather a series of sequential decisions. This aspect of triage was ignored by Hupert et al in their model. A single triage decision is unreliable, even when made by experienced physicians. ${ }^{4}$ Triage accuracy comes from repetition and reiteration of the triage process as the casualty is moved between service points in the hospital. This redundancy permits a 


\section{FIGURE 1}

\section{Relationship of overtriage rate to critical mortality rate in 10 (1969-1995) vs 4 (2001-2007) terrorist events. Note shift of curve to left.}

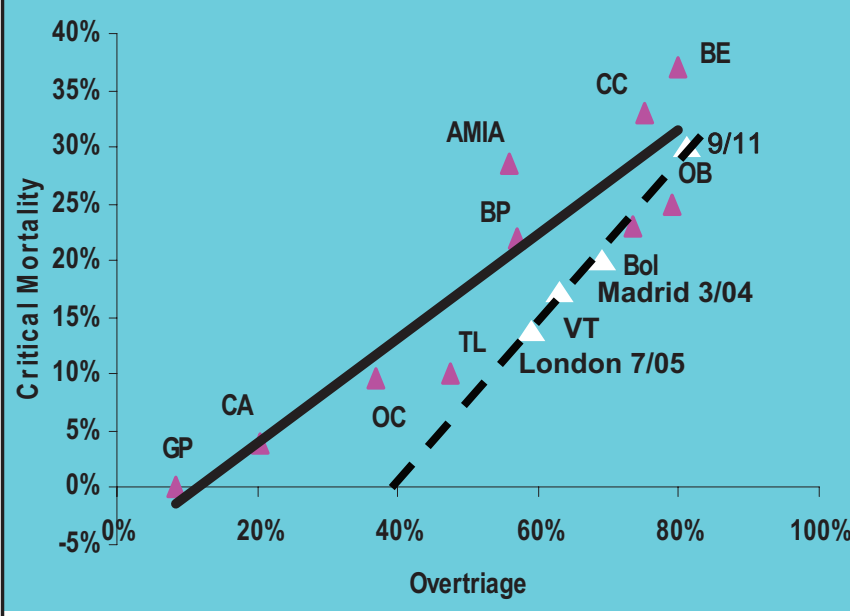

$\mathrm{GP}=$ Guildfordpubs; $\mathrm{CA}=$ Craigavon; $\mathrm{OC}=$ Oklahoma City bombing; $\mathrm{TL}=$ Tower of London; $\mathrm{BP}=$ Birmingham pubs; $\mathrm{Bol}=$ Bologna; $\mathrm{AMI} \mathrm{A}$ $=$ Buenos Aires; $\mathrm{OB}=$ Old Bailey; $\mathrm{CC}=\mathrm{Cu} \mathrm{Chi}$; $\mathrm{BE}=$ Beirut; $\mathrm{VT}=$ 2007 Virginia Tech shooting. Adapted from Frykberg ER. Medical management of disasters and mass casualties from terrorist bombing: how can we cope? J Trauma. 2002;53:201-212.

greater tolerance of triage errors as they are caught and corrected at successive levels of care.

Is this kind of modeling study helpful? Yes. Despite some obvious limitations, the article generates a fruitful discussion surrounding a core question in disaster medicine: how does overtriage influence critical mortality? It also emphasizes more broadly the critical role that triage plays in determining outcome, and therefore, the importance of intensive training in triage among medical providers who may be confronted by the unique challenges of mass casualty care. In analyzing its flaws, we are reminded that "planning should take into consideration how people and organizations are likely to act, rather than expecting them to change their behavior to conform to the plan." 5 In essence, the article becomes a call for more research in triage, focused on an insightful combination of theoretical modeling with evidence from real-life experience.

\section{About the Authors}

Dr Armstrong is Assistant Professor of Acute Care Surgery, University of Florida; Dr Hammond is director of the trauma center at the Robert Wood Johnson Medical School, University of Medicine and Dentistry of New Jersey; Dr Hirshberg is Associate Professor of Surgery, Baylor College of Medicine; and Dr Frykberg is Chief, Division of General Surgery, University of Florida.

Correspondence and reprint requests to Dr John H. Armstrong, 1600 SW Archer Rd, Box 100286, Gainesville, FL 32610-0286 (e-mail: john.armstrong@surgery. ufl.edu).

Received for publication December 5, 2007; accepted December 6, 2007.

\section{Authors' Disclosures}

The authors report no conflicts of interest.

ISSN: 1935-7893 (C) 2008 by the American Medical Association and Lippincott Williams \& Wilkins.

DOI: $10.1097 / D M P .0 b 013 e 31816476 c 0$

\section{REFERENCES}

1. Hupert N, Hollingsworth E, Xiong W. Is overtriage associated with increased mortality? Insights from a simulation model of mass casualty trauma care, Disaster Med Public Health Preparedness. 2007;1:S14-S24.

2. Auf der Heide E. The importance of evidence-based disaster planning. Ann Emerg Med. 2006;47: 34-49.

3. Gutierrez de Ceballos JP, Turegano-Fuentes F, Perez-Diaz D et al. March 2004: the terrorist bomb explosions in Madrid, Spain —an analysis of the logistics, injuries sustained and clinical management of casualties treated at the closest hospital. Crit Care. 2005;9:104-111.

4. Ashkenazi I, Kessel B, Khashan T et al. Precision of in-hospital triage in mass-casualty incidents after terror attacks. Prehosp Disaster Med. 2006; 21: 20-23.

5. Quarantelli EL. Organization Behavior in Disasters and Implications for Disaster Planning, Report series 18. Newark, DE: Disaster Research Center, University of Delaware; 1985

\section{Response to Armstrong et al}

Nathaniel Hupert, MD, MPH, Eric Hollingsworth, BS, and Wei Xiong, PhD

We are pleased that the thought leaders and originators of this line of research consider our article $^{1}$ to be a useful contribution to ongoing discussions about improving mass casualty trauma care. Our approach focused on the tripartite, dynamic relationship among patient selection resulting from triage decisions, trauma system treatment capability, and time-dependent mortality. Our main finding is that, for most mass casualty incidents, triage accuracy has less impact on outcomes than does the relative proportion of critical casualties to treatment capability, with the corollary that focusing on the rate of overtriage (ie, getting triage "wrong" in the direction of overcrowding) may obscure other drivers of critical outcomes.

As noted by Armstrong et $\mathrm{al}^{2}$ in this issue, our model did produce "a positive correlation between overtriage and critical mortality when the number of noncritical casualties increases" but this relationship is both nonlinear and dependent on the ratio of critical casualties to treatment bays. For all of its limitations, this model represents a conceptual framework that begins to reflect the complex relationships among actions, resources, and patient outcomes, and we will continue our efforts to improve its fidelity to the realities of trauma care in both the field and hospital settings.

\section{About the Authors}

All of the authors are with the Department of Public Health, Weill Medical College, Cornell University.

Address correspondence and reprint requests to Dr Nathaniel Hupert, Department of Public Health, Weill Medical College, Cornell University, 411 E 69th St, Room KB-313, Box 74, New York, NY 10021 (e-mail: nah2005@med.cornell.edu). 